\title{
Smart Technologies in the future housing constructions
}

\author{
Mohammadali Heidari, Erfaneh Allameh
}

Art University of Isfahan, Isfahan,Iran

\begin{abstract}
Researchers envision a future information society stemming from ubiquitous computing and intelligent environments. To a large extent, an ambient intelligent home called smart home no longer is science fiction and is technologically feasible. But reviewing the current state of the field shows that application of the smart home in real life and in the future housing constructions is still lacking; largely because the investigation of smart homes is limited to the domain of technical issues. But applying smart technologies in a home environment affects the way people live inside and outside of their home and shapes a new lifestyle. When the way of living changes the conditions of the dwelling change accordingly. However, usually the technology is added after the spatial design in the final design stage by the installation expert. Hence, a mismatch between the user demands and the smart home possibilities has been occurred.

In this paper, we turn this process around; the smart technologies are accommodated by spatial design and shape a smart home. Specifically, we model the new spatial characteristics of smart homes based on users preferences. The model is based on the assumption that different individuals and households have different spatial preference of smart homes due to having various characteristics, lifestyles, and needs. A Bayesian Belief Network (BBN) is used for the modeling. It estimates the probabilities of choosing spatial characteristics of the smart home among different users with various sociodemographic characteristics. The spatial characteristics which are going to be predicted relate to the public-private layout of smart homes.

By determining new spatial organizations based on users` preferences, smart homes can practically provide spaces that respond to the users` needs in real life. Proper integration of technology with space and adjusted spatial conditions are vital for the accomplishment of smart homes and improving the users`acceptance.
\end{abstract}

(C) 2018 The Authors. Published by Diamond Congress Ltd., Budapest University of Technology and Economics Peer-review under responsibility of the scientific committee of the Creative Construction Conference 2018.

Keywords: housing construction, smart technologies, technology-space integration, users` preferences;

\section{Introduction}

Applying new smart technologies in the current houses change many of the current living patterns of people. The lifestyle study of smart homes indicates that many of the daily living patterns are going to be changed by applying smart technologies in houses. But the home is meant primarily to support the activity "to live". When living patterns change because of embedding the new technologies, conditions of the home need some redefinitions accordingly. In this paper, we aim to define optimal spatial layout of smart homes, that is, adapting interior spaces of a smart home to the users` needs and preferences in the real life and establishing the highest functionality of the applied smart technologies. But the main focus of this paper is given only to the public-private layout of smart homes.

Thanks to applying smart technologies in the domain of a home, the private zone of a smart home is going to be used less than an ordinary homes. People typically use bedrooms of the smart home only for sleeping. Instead, they prefer to do most of their daily activities, even working, in public areas and use the smart facilities. Thus, when activities

Corresponding author: Mohammadali Heidari email: m.heidari@aui.ac.ir 
move from the bedroom and take place in other spaces, bedrooms seem to be required less. There are some existing literatures, which claim that bedrooms in future houses will be replaced by cozy islands in the home layout. Certain rooms in the home will likely disappear and will be replaced by multifunctional spaces (Living Tomorrow lab [8], Horx [4]). Moreover, the main advantage of ambient intelligent technology in the smart home is improving the level of flexibility in the ways of doing activities. Any corner in the smart home is suitable for working or doing tele activities thanks to ICT and AmI technologies. Due to these changes, an architecturally distinct area is no longer required and the separation of work space and living space is increasingly broken down and rearranged by "blurring boundaries" (Leonard et al. [7]). In such a scenario, some granted boundaries between spaces may dissolve. The former physical separation of work or personal activities in the private spaces and other daily activities in the public spaces of the home does no longer apply in the same way. The home allows users to experience open and livable flow in the multifunctional smart zones rather than separate rooms. Hence, public- private layout of a smart home needs some redefinitions regarding to the proportion, the integration and the level of flexibility between the public and private zone. For defining the optimal spatial layout of the public-private zone, we conduct an experimental research. In fact, we investigate the new layout based on users` preferences. In the following, the method for eliciting users` preferences and accordingly estimating the optimal spatial layout are discussed.

\section{The experiment}

\subsection{Implementation of the experiment}

Smart homes are not still widely being applied in the housing industry and are mostly applied in a living lab environment. Implanting an experimental research on smart homes could be challenging in this regard, since the majority of the respondents does not have enough comprehension of the subject. In the implementation of the experiment, we take this issue into consideration. We design the experiment in a way that respondents not only explore how a smart home will look like, but also can interact with it. In fact, we first improve the experience of respondents from the smart homes and then elicit their preferences through some tasks instead of asking some direct questions. Through the tasks, users can rearrange spatial layout of a smart home. In fact, to have a more trustworthy experimental approach, we are going to explore how people make a smart home. Through their design choices, we elicit the spatial preferences of different target groups.

\subsection{Design of the experiment}

We use our developed prototype application as a platform for applying the experiment (Heidari et al. [3]). The virtual smart home presented in this prototype consists of several smart technologies, namely, smart walls, smart kitchen table, smart private zone, and smart furniture. We design the experiment with three steps, which are executed in the virtual smart home by users in the real-world: Step 1) the initial questionnaire, Step 2) a virtual tour through the smart home, Step 3) spatial arrangement. These three steps are depicted in Figure 1.

The experiment starts with an initial questionnaire with multiple sections, in which we ask some questions about the respondents`characteristics (step1). Then, respondents take a virtual tour though the smart home environment and watch several movies about smart technologies in a smart home (step2). In this part, we introduce a smart home to the respondents, let them explore the spaces and the embedded smart technologies in it, and accordingly improve their experience of the smart homes.

Step 3 is the spatial arrangement, in which respondents make multiple design choices for different parts of a smart home and design their preferred home layout. For each part, respondents explore multiple design alternatives and then choose one of them. In fact, a respondent can explore all the possible combinations until reaching to the final decision and selecting the most preferred layout. The spatial preferences can be elicited from the selected layout. While the design alternatives cover different parts of a smart homes, we discuss only the public-private layout in this paper. The possible combinations for the public-private layout are: a) small, integrated private, no flexible room, b) small, integrated private, flexible room, c) medium, semi-separate private, no flexible room, d) medium, semi-separate private, flexible room, e) large, separate private, no flexible room. All the alternatives related to the public-private layout are depicted in Figure 2. As depicted, the alternative (e) which has the largest bedroom cannot offer the extra flexible space. Since having a flexible space requires spatial reduction of the private zone. This alternative defines a large private zone with equal proportion among private and public zone, fixed boundaries and no opportunity for having flexibility. Hence, it is the most similar layout to the current houses. 


\begin{tabular}{|c|c|c|c|c|}
\hline Open plan layout & Flexible/integrated layout & Separated & Separated/flexible & Separated \\
\hline $\begin{array}{l}\text { Smallest private zone } \\
\text { No flexible room } \\
\text { Largest public zone }\end{array}$ & $\begin{array}{l}\text { Smallest/flexible private } \\
\text { zone } \\
\text { flexible room } \\
\text { Largest/flexible public } \\
\text { zone }\end{array}$ & $\begin{array}{l}\text { Middle private zone } \\
\text { No flexible room } \\
\text { Large public zone }\end{array}$ & $\begin{array}{l}\text { Middle private zone } \\
\text { flexible room } \\
\text { Small/flexible public zone }\end{array}$ & $\begin{array}{l}\text { Largest private zone } \\
\text { No flexible room } \\
\text { Smallest public zone }\end{array}$ \\
\hline
\end{tabular}

Fig 1. An illustration of the choice alternatives for the public-private layout

\subsection{Conducting the experiment}

The experiment was Internet-based with the sample size of 250 respondents. From all of the respondents, 48.8 percent were single incomes, 41.7 percent were dual incomes, while the remainder were not working. 76 percent of the respondents were less than 34 years old (young), 20.5 percent were between 35 to 54 years old (middle aged) and the remainder were above 55 (elderly). This sample contains international respondents mainly from Iran, the Netherlands and other countries.

\section{Model Specification}

In the this paper, we aim to define the optimal public-private layout of smart homes, which is adapting the spatial aspects of these zones to the users` needs and preferences in the real life and establishing the highest functionality of the applied smart technologies.

Hence, a Bayesian Belief Network (BBN) is used to estimate and formulate the relations between the variables that directly and indirectly influence on the users` spatial preferences of smart homes. A BBN is composed of a set of variables, connected by links to indicate dependencies. It also contains information about relationships between the variables. For each variable, a conditional probability table (CPT) is provided, which quantifies how much a variable depends on its parents (if any). The belief network can be used for measuring users` spatial preferences since we can use the cause-effect relations between nodes to represent causal relations in the preference structure. Orzechowski [10] has proven in the past, that a learning based approach like the Bayesian Belief Networks allows estimate users ${ }^{`}$ preferences in a choice model. Using the Bayes rule, the posterior belief $\mathrm{p}(\mathrm{A} \mid \mathrm{B})$ can be calculated by multiplying the prior belief $\mathrm{p}(\mathrm{A})$ by the likelihood $\mathrm{p}(\mathrm{B} \mid \mathrm{A})$ that $\mathrm{B}$ will occur if $\mathrm{A}$ is true.

$$
P(A / B)=\frac{P(B / A) \cdot P(A)}{P(B)}
$$

The Bayes' rule is helpful in many situations where we want to compute $\mathrm{p}(\mathrm{A} \mid \mathrm{B})$ but we cannot do so directly (Orzechowski [10]). It is common to think of Bayes' rule in terms of updating the belief about a hypothesis A in the light of new evidence B. In the following, we argue how we apply this method to predict spatial preferences (as hypothesis A) in the light of new evidences of the users ' characteristics (e.g. working status and age) and other external influencing factors such as size of the smart home.

The estimation of a BBN requires, as a first step, the process of learning the network structure of the data, and then estimation of the conditional probability tables (CPT) (Cheng et al. [2]). The constructed BBN (Figure 2) is compiled and displayed in the Netica (Norsys Software Corp [9]).

It is going to estimate optimal spatial layouts for the public-private zones of the smart homes. The presented BBN in Figure 2 is part of a huge network with multiple nodes indicating more spatial aspects (e.g. Smart living room layout or smart kitchen layout) and more influencing factors on users' preferences (e.g. Users' characteristics and lifestyles). In this paper, a particular emphasize has been placed only on the most important influencing factors on the preference for the public-private layout. 


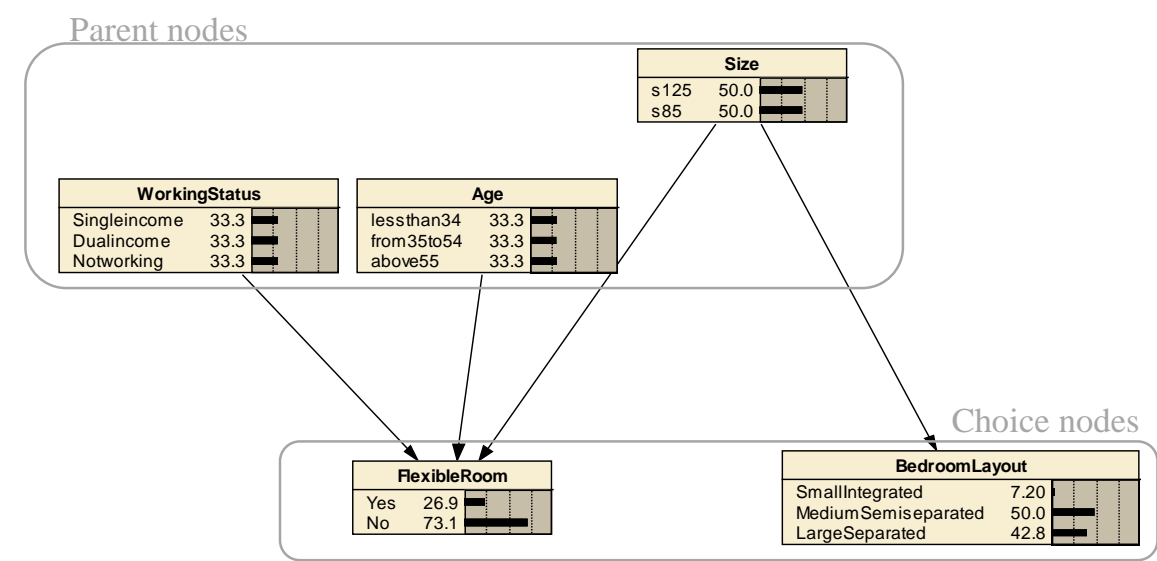

Fig 2. The constructed BBN estimating users` preferences of the public-private layout of a smart home based on their characteristics and the size of the smart home

\section{Results}

\subsection{Spatial preferences in different sizes of smart homes}

It is assumed that the size of smart home has an important effect on users ' preferences. While smart technologies can easily be added to a large sized house without any cost concerns, applying smart technologies in smaller sized houses should be based on economic feasibility. Due to the matter of cost, there will be a low chance for smart homes to be accepted by middle or low incomes, especially if the applied smart technologies cannot really improve the quality of space. Hence, spatial design of smart homes with a limited size would be so challenging if smart homes want to be targeted for a wide range of target groups. In this study, we focus on the two sizes of $125 \mathrm{~m}^{2}$ and $85 \mathrm{~m}^{2}$ to evaluate the effects of limiting the size on the users`spatial preferences. $125 \mathrm{~m}^{2}$ is considered as a normal sized smart home and 85 $\mathrm{m}^{2}$ is considered as a small scaled smart home.

Table.1 probability estimation of the BBN for users` preferences of the public-private layout in different sizes

\begin{tabular}{l|ll}
\hline & Size125 & Size85 \\
\hline Bedroom Layout & $\mathbf{1 1 . 3 7 8}$ & 3.0173 \\
Small/Integrated & 40.97 & $\mathbf{5 9 . 0 4 7}$ \\
Medium/Semi-separated & $\mathbf{4 7 . 6 5 2}$ & 37.936 \\
Large/Separated & & \\
\hline Flexible Room & 20.342 & $\mathbf{3 3 . 5 5 5}$ \\
Yes & $\mathbf{7 9 . 6 5 8}$ & 66.445 \\
No & & \\
\hline
\end{tabular}

As represented in Table 1, the preference for the flexible room goes down and the preference for the large, separated bedroom layout goes up in a $125 \mathrm{~m}^{2}$ smart home; because, there is enough space in this size to apply smart technologies only in the public zone, while keeping the private zone as large and separated as it is (Figure 2, combination e). But according to the estimations in Table1, despite of such a kind of high preference for the large, separate private zone in this size, the majority of people prefer to minimize and integrate the private zone of the smart home; the total probability of choosing the small, integrated bedroom layout and the medium, semi-separated layout (52.348 \%) is higher than the probability of choosing the large, separated bedroom layout. More specifically, the preference for the small, integrated bedroom layout (Figure 2, combination a) significantly increases in this size in comparison with the $85 \mathrm{~m}^{2}$ smart home. To conclude, although there is a risk for users to fallow the multi room layout of thier current houses in a $125 \mathrm{~m}^{2}$ smart home and do not modify the private zone, the hypothesis of minimizing the private zones and prefering a more open plan layout is valid in this size. In contrast, by decreasing the size of the smart home into 
the $85 \mathrm{~m}^{2}$, the preference for the medium, semi-separate bedroom layout with the extra flexible room increases (Figure 2 , combination $\mathrm{d}$ ). This result is an indication that having a large living room by minimizing the bedrooms and making them flexible is the priority in people`s design decision for a small scaled smart home. In this size, applying smart technologies, mainly affects the private zone of the Smart home. The majority of people prefers to reduce the size of the private zone and accordingly have a larger living room. The increasing preference for the flexible room is due to the reason that the flexible room can be completely open to the living room whenever it is not needed and therefore it can create a larger living room. To conclude, applying Smart technologies in small scaled houses can really open up inessential separations and makes more open space and flexible layout for these houses.

\subsection{Spatial preferences based on users`working status and age}

According to the BBN, the working status and the age of people influence on their preferences for the level of flexibility between the public zone and the private zone in smart homes. Figure 3 shows how these two nodes change the probabilities of choosing the flexible room.

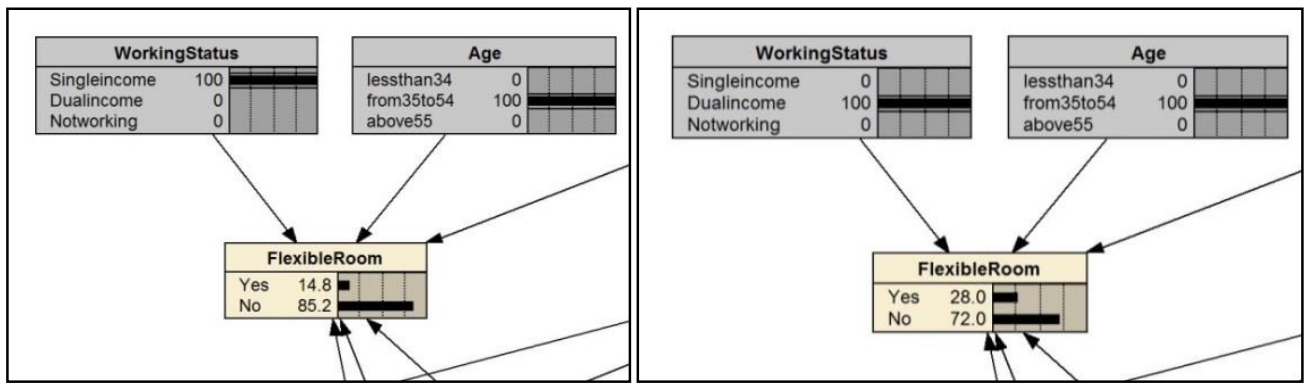

Fig.3 A screen shot of the BBN showing the flexible room node with its parent nodes of working status and age

In this part, more detailed exploration is given about the variation of this general preference among people with different age and working status. According to Table 2, the preference for having the flexible room is increased by increasing the age. While young people in all of the working status have the least preference for the flexible room, elderlies have the highest preferences for it. The reason may rely on the fact that elderlies need to manage their privacy more than other people and the flexible room can help them in this regard. People, who not-work (retired, housewives and etc.), have an increasing preference for the flexible room. They usually spend a lot of time at home. Hence, flexibility of spaces is more important for them compared to people, who work out of the home. Moreover, dual incomes have a higher preference for the flexible room than single incomes. Dual incomes usually have a busier lifestyle than single incomes. They usually have a tight schedule while they are at home. Hence the flexible room can help them to reduce the possible conflicts among their activities. In conclusion, while the general estimations indicated that the flexible room is not largely preferred in smart homes, case analysis on the BBN reveal that elderlies, notworking people, and dual incomes are the potential target groups for the spatial flexibility inside the smart homes. They are people, who need different ranges of privacy and a flexible room helps them to adjust the privacy of spaces according to their different types of activities.

\begin{tabular}{|c|c|c|c|}
\hline & Single inc, young & Single inc, middle aged & Single inc, elderly \\
\hline \multirow{4}{*}{$\begin{array}{l}\text { Flexible Room } \\
\text { Yes } \\
\text { No }\end{array}$} & & & \\
\hline & $\underline{10.296}$ & 14.8 & 26.231 \\
\hline & 89.704 & 85.2 & 73.769 \\
\hline & Dual incomes, young & Dual inc, middle aged & Dual inc, elderly \\
\hline \multirow{4}{*}{$\begin{array}{l}\text { Flexible Room } \\
\text { Yes } \\
\text { No } \\
\end{array}$} & & & \\
\hline & $\underline{20.735}$ & 27.961 & 43.526 \\
\hline & 79.265 & 72.039 & 56.474 \\
\hline & Not-working, young & Not-working, middle aged & Not-working, elderly \\
\hline \multicolumn{4}{|l|}{ Flexible Room } \\
\hline Yes & $\underline{22.63}$ & 30.21 & 46.147 \\
\hline No & 77.37 & 69.79 & 53.853 \\
\hline
\end{tabular}




\section{Conclusion}

The most common concept of space in current houses is its categorization by functions, such as sleeping rooms, working room and living room (Junestrand [6]) that can be traced to the "form follows function" design philosophy by American architect Louis Sullivan`s established more than a century ago. But the gained results in this paper revealed that smart technologies have the possibility to break down many of the boundaries, separations, and spatial limitations in the internal spaces of houses. Applying smart technologies decreases the occupied area and the separation of the private zone at homes. The private zone becomes more flexible in smart homes. Hence, the public zone becomes larger and more integrated to the private zone. Such a kind of change from the multi-roomed layout to a more flexible, multifunctional open plan layout in smart homes matches better with the new living patterns of an information society. However, these spatial modifications are mainly applicable for target groups, who really can be benefited from the smart technologies. Only if people really utilize the smart technologies in their daily life, they try to modify the space in order to achieve the full functionality of the smart technologies. For this reason, dual incomes more than single incomes, elderlies, and middle aged people more than young people, inhabitants of small scaled houses more than people, who live in larger houses, prefer a flexible and open layout in the smart homes. In conclusion, by increasing the age and numbers of people who work at home or by decreasing the size the need for applying the smart technologies is increased and accordingly the preference for having more flexibility and integration in the public and private zone of a smart home is increased. But if people do not really need the smart technologies and do not apply them in their daily life, there is a high probability that they prefer the most similar layout to the layout of their current house; because people generally get inspired from the spatial layout of their current house.

To conclude, eliciting spatial preference of different target groups and apply them in the smart home design broaden the domain of smart homes from a technology-driven industry to the future housing industry and real estate. There is a great opportunity to apply the smart technologies not only in living labs, luxurious houses, or assisted houses, but also in more common types of housing, like the small and medium sized apartments.

\section{References}

[1] Aarts, H,.; Verplanken, B,; Knippenber, A.: Predicting Behavior From Actions in the Past: Repeated Decision Making or a Matter of Habit?, In: Journal of Applied Social Psychology, 1998, 28, 15, 1355-1374

[2] Cheng, J., Bell, D., Liu, W., Learning Bayesian networks from data: an information-theory based approach. Artificial Intelligence, 2002, $137,43-90$.

[3] Heidari, M.; Allameh, E.; De Vries.; B. Timmermans,; Jessurun, J.; Mozaffar, F.: Smart-BIM virtual prototype implementation. In: Automation in Construction, 2014, 39, 134-144

[4] Horx, M., "Smart Home: Home of the Future", The Future Evolution House Vienna. Available at: http://www.zukunftshaus.at/English/PressInfo.aspx, 2009 .

[5] Jullisson, E.A.; Karlsson, N.; Garling, T.: Weighing the past and the future in decision making. In: Journal of Cognitive Psychology, 2005, $17,561-575$

[6] Junestrand, S. Being private and public at home, An architectural perspective on video mediated communication in Smart Homes, KTH Doctoral thesis, Stockholm, Sweden, 2004.

[7] Leonard, L. and Thorns C. D. On-Line Workers Working From Home, Proceedings of the SAANZ, 2006.

[8] Living Tomorrow Lab. Belgium: http://events.livingtomorrow.be/EN/Rondleidingen.aspx, 2007.

[9] Norsys Software Corp. Netica 3.17 for MS Windows. Vancouver, Canada, 2006.

[10] Orzechowski, M. Measuring housing preferences using virtual reality and Bayesian belief networks. Eindhoven: Technische Universiteit Eindhoven, 2004. 